\title{
Synthesis of Bistable Periodic Structures Using Topology Optimization and a Genetic Algorithm
}

Department of Mechanical Engineering, Michigan State University, East Lansing, Ml 48824-1226
A formulation for the automatic synthesis of two-dimensional bistable, compliant periodic structures is presented, based on standard methods for topology optimization. The design space is parametrized using nonlinear beam elements and a ground structure approach. A performance criterion is suggested, based on characteristics of the load-deformation curve of the compliant structure. A genetic algorithm is used to find candidate solutions. A numerical implementation of this methodology is discussed and illustrated using simple examples. [DOI: 10.1115/1.2338576]

\section{Introduction}

A bistable compliant structure has two stable equilibrium configurations when unloaded. Once such a structure reaches one of its stable configurations, it remains there unless it is provided with enough energy to "climb" out of an energy well that leads into the other stable configuration. This feature of bistable structures can be advantageous in designing a variety of mechanical devices, such as switching devices in microelectromechanical systems (MEMS), relays and valves. We use the term bistable periodic structure to refer to an arrangement of interconnected bistable structures that tile a plane periodically. The load need not be periodic and the tiling need not be infinite. This paper discusses a computational strategy to design bistable periodic structures using topology optimization.

An arrangement "in series" of bistable structures that tile a plane periodically can achieve larger overall displacements while keeping both the actuating force and the deformation of each structure small. This is of particular interest in design of microsensors or actuators, where the amount of energy available to produce a desired motion may be small. Furthermore, developing a methodology for design of microscale periodic arrangements of such bistable structures is a first step towards designing microstructures that behave as bistable materials, such as, for example, shapememory alloys and the "negative stiffness" systems discussed in Ref. [1]. Such materials have interesting applications as actuators and sensors, but have drawbacks associated with, e.g., response time, the amount of energy required to switch states, and the ease with which this energy can be introduced into the system. A microscale periodic arrangement of bistable structures may be designed to have tailor-made energy storage properties, designed to meet criteria specific to each application, perhaps overcoming the limitations of known, currently available materials. While this is admittedly some distance into the future, this is another important motivation for this work.

A typical example of a bistable compliant structure is a bistable compliant mechanism (e.g., see Refs. [2-8]). Such mechanisms typically rely on strain energy storage to gain bistable behavior. They are made of flexible members, which produce motion and at

${ }^{1}$ Corresponding author.

Contributed by the Mechanisms and Robotics Committee of ASME for publication in the Journal of Mechanical Design. Manuscript received August 1, 2005 final manuscript received December 28, 2005. Review conducted by Larry L. Howell. Paper presented at the ASME 2005 Design Engineering Technical Conferences and Computers and Information in Engineering Conference (DETC2005), September 24-28, 2005, Long Beach, CA. the same time, store energy. Compliant mechanisms in general (e.g., see Ref. [9]) offer several advantages, especially in design of MEMS, as discussed in Ref. [10], and have been the subject of extensive research. Particularly relevant to our effort is previous work where a topology optimization strategy is used to synthesize compliant mechanisms, e.g., as discussed in Refs. [11-14].

A distinguishing feature of any bistable compliant structure is the shape of its strain energy curve. Figure 1 shows a qualitative description of the variation of strain energy with strain for a typical bistable compliant structure. The function is nonconvex. The strain energy curve has three critical points - two minima (points $C$ and $G$ ) and one maximum (point $E$ ). When the system is unloaded, and under small loads, the structure will operate in and around one of the two minima, corresponding to one stable configuration. Transition from one configuration to the next requires the addition of sufficient energy to jump over the small maximum and over into the neighborhood of the other minimum. An external load provides this activation energy in the form of external work and switches the structure from one stable configuration to the other. Once the new state is reached, again the structure will operate in and around this configuration, for small enough inputs. As indicated in Fig. 1, there are other characteristic points on the strain energy curve, which are described in detail in Sec. 4.2. The shape of the strain energy curve around these points gives quantitative measures of the bistability. We will use these points to produce a measure of performance that can guide an eventual methodology for optimization of bistable periodic structures.

The design of compliant mechanisms typically follows one of two principal approaches. The kinematics approach produces a structure composed of small flexible pivots and relatively rigid links (e.g., as in Refs. [5,7]). This approach results in lumped compliance, such as locally deforming flexural joints. The structural topology optimization approach is based on the methodology for topology optimization of structures introduced by Bendsøe and Kikuchi [15] and uses either a continuum modeling based on plane elasticity (e.g., Refs. $[12,16]$ ) or a ground structure approach that relies on slender members (beams and bars, e.g., Refs. $[13,17-19])$. Genetic algorithms have been used in this context, e.g., by Tai and Chee [14] and Saxena [20].

In this work we investigate the automatic topology synthesis of two-dimensional bistable compliant, periodic structures using a ground structure topology optimization approach. The presentation will proceed as follows. In Sec. 2 we introduce a concept for a bistable structure that can be repeated periodically to tile the plane. This concept is used simply to highlight basic features that one may expect to find in the layout of these structures. Section 3 


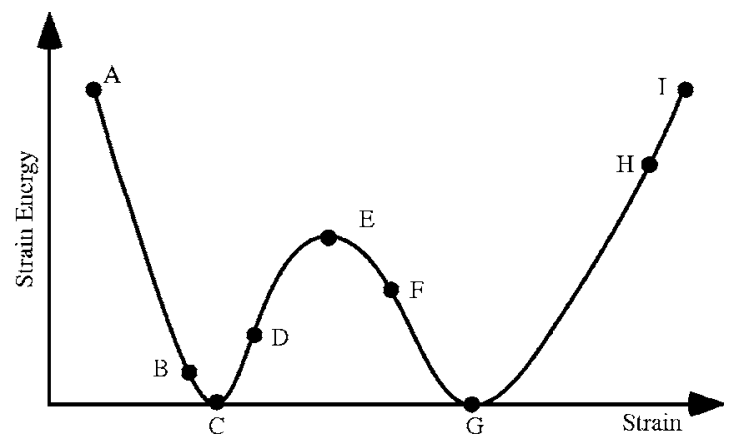

Fig. 1 Strain energy versus strain in a typical bistable structure

describes the finite element model we used for analysis of compliant bistable structures. A possible topology optimization problem formulated to synthesize a compliant bistable structure is described in Sec. 4. Three examples illustrate the methodology in Sec. 5. Conclusions and a discussion of opportunities for further work close the presentation.

\section{An Example of a Bistable Periodic Structure}

In this section we introduce a design concept that can be repeated periodically to tile the plane and form a bistable periodic structure. This concept is used to highlight basic features of these structures that may be used later in deciding how to build a ground structure for topology optimization.

In Qiu et al. [21], the authors present a monolithic mechanically bistable mechanism that uses no latches, no hinges, and no residual stress. The typical implementation of this mechanism involves two curved, centrally clamped parallel beams of uniform cross-section, labeled "double curved beams." Figure 2 shows a simplified double curved beam bistable mechanism, where each of the curved beams (shown as dashed lines) is approximated by two straight beams. This simple concept may be used to build a twodimensional bistable structure, in this case, using four such bistable mechanisms, as shown in Fig. 3. In the figure, one of the four component bistable mechanisms is encircled by a dashed line and shown alone in Fig. 4. The original central clamp (the dark, vertical line in Fig. 2) is replaced by a stiff triangular structure in Figs. 3 and 4. This allows the incorporation of two input points, where loads are applied on each side of the structure. Qiu et al. [21] report that a clamped double-beam structure (Fig. 2) is not bistable if its geometry is not carefully selected. The authors demonstrate that a uniform cross-section single beam structure is not bistable. They also show that a varying cross-section single beam can be made bistable by thickness modulation; however, the stress state in the modulated beam is several times higher than that in a uniform cross-section beam. Here we attempt to take advantage of both types of bistable structures-uniform cross-section doublebeam and varying cross-section single-beam, by using a varying cross-section double-beam structure. Accordingly, the beams here are thickness modulated, i.e., the cross-sectional area of either beam in a double-beam structure is allowed to vary along its length to provide more control on the bistability characteristics of

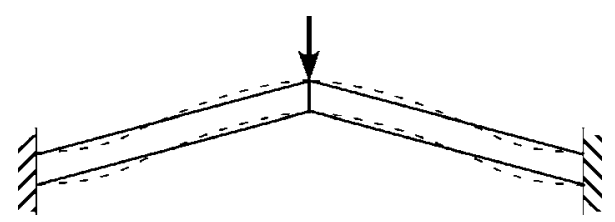

Fig. 2 Simplified double curved beam bistable mechanism

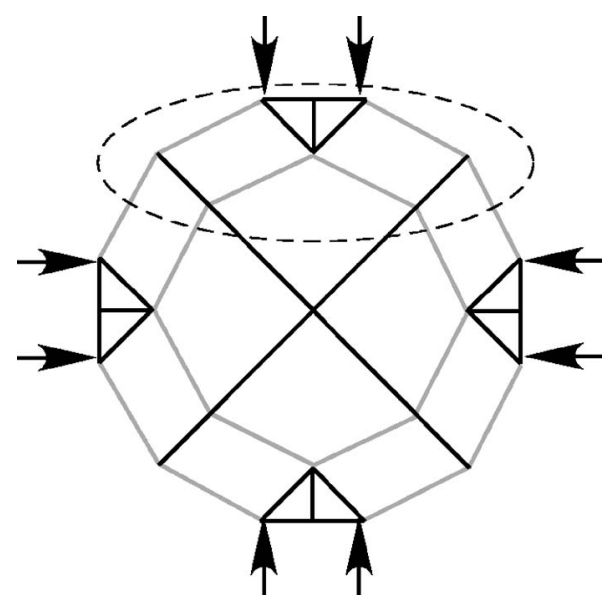

Fig. 3 A bistable structure (first stable configuration) based on four double curved beam substructures

the structure. A simple way of implementing a thickness modulation considered here is to use beams of two different crosssectional areas.

Bistability is achieved by designing the structure such that some of the members buckle or "snap." In the structures shown in Figs. 3 and 4, members which may snap are shown as light gray lines. Members shown as dark lines are stiffer, stiff enough to provide clamping support to the snapping beams. The configuration shown in Fig. 3 corresponds to the first equilibrium configuration of the structure. When loaded as shown by a load of sufficient magnitude, the structure moves into its second equilibrium configuration, shown in Fig. 5.

The bistable structure shown in Fig. 3 can be repeated periodically to tile a plane, joining each unit together via rigid connectors (shown as solid rectangles in Fig. 6). Two stable equilibrium configurations of such structure are shown in Fig. 6. The dashed box shows the fundamental cell of the periodic structure.

The solution proposed in Fig. 3 was generated by analogy, using the double curved beam structure as guide. We are seeking a computational strategy that may allow us to synthesize other such structures and meet some prescribed performance specification, e.g., match certain desirable features of a strain energy curve or a load deformation diagram. One such strategy based on topology optimization is discussed in the following sections.

\section{The Analysis Model}

The structure is analyzed using nonlinear, corotational Timoshenko beam elements and a total Lagrangian formulation (a detailed formulation is given in Ref. [22], p. 219.) The analysis is quasi static and all inertial forces are ignored. At equilibrium

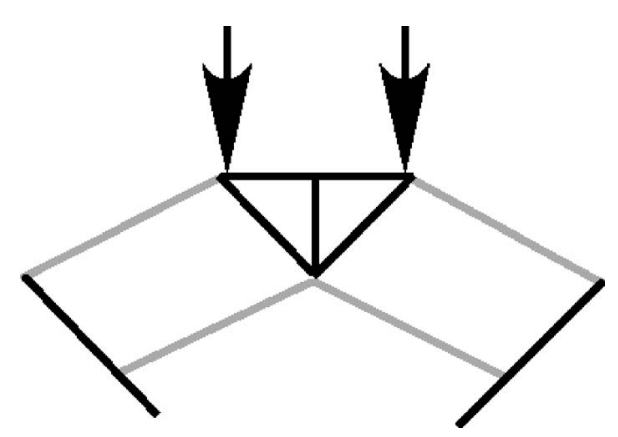

Fig. 4 A substructure of Fig. 3 that is a bistable mechanism 


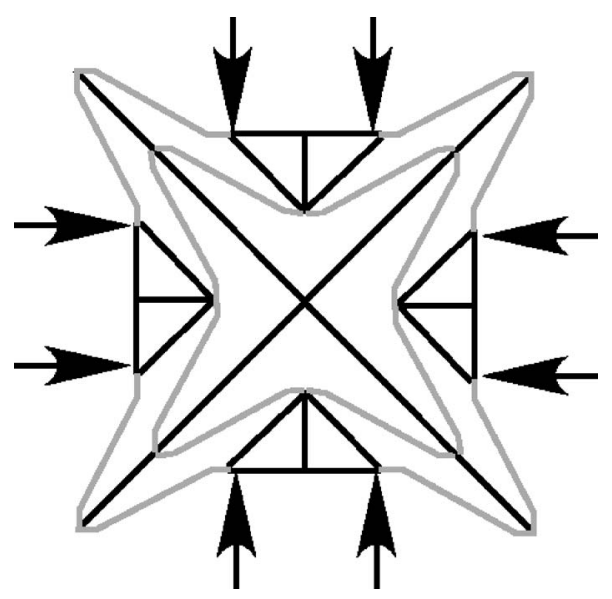

Fig. 5 A bistable structure (second stable configuration) based on four double curved beam substructures

$$
\mathbf{g}(\mathbf{x})=\mathbf{f}_{\text {int }}(\mathbf{x})-\mathbf{f}_{\text {ext }}(t)=0
$$

where

$$
\mathbf{f}_{\text {int }}(\mathbf{x})=\frac{\partial \Lambda}{\partial \mathbf{x}}
$$

is the internal force, $\Lambda$ is the total strain energy, $\mathbf{x}(t) \in \mathfrak{R}^{n}$ represents the configuration of the structure at time $t$ under an external force $\mathbf{f}_{\text {ext }}(t)$ and $n$ is the total number of degrees of freedom. A Newton-Raphson-like scheme is used to find a solution to (1). At the $i$ th iteration step the scheme produces

$$
\mathbf{x}_{i+1}^{t}=\mathbf{x}_{i}^{t}-\left[\frac{\partial \mathbf{g}}{\partial \mathbf{x}}\right]_{\mathbf{x}=\mathbf{x}_{i}^{t}}^{-1} \mathbf{g}\left(\mathbf{x}_{i}^{t}\right)
$$

where $\mathbf{x}_{0}^{t+1}=\mathbf{x}^{t}$. In (3), $\partial \mathbf{g} / \partial \mathbf{x}$ is the tangent stiffness matrix. $\mathbf{x}^{t}$ represents the solution to the equilibrium equation (1) at time $t$.

Simulation results were validated using the commercial finite element package ABAQUS using the element "b21," a beam ele- ment. As the model consists only of beam elements, joint rigidity may be overestimated, when compared to a full $2 \mathrm{D}$ elasticity model (e.g., one that uses quadrilateral finite elements). This drawback can be overcome by the modulation of the crosssectional area, as discussed in the next section.

\section{Optimization of the Topology}

4.1 The Ground Structure and Design Variables. The formulation used here is similar to a classical ground structure approach in truss topology optimization (e.g., Refs. [23,24]). In a typical ground structure approach, design variables are crosssectional areas $A_{\alpha}$ of each member (bar) in the ground structure, where $\alpha=1,2, \ldots, n_{b}$ and $n_{b}$ is the total number of members in the ground structure. The ground structure contains enough members and possible connections to include, as a subset, a reasonable number of design alternatives. The moment of inertia of the beam $I_{\alpha}$ is assumed to be proportional to the square of the beam's crosssectional area, i.e.,

$$
I_{\alpha}=c A_{\alpha}^{2}
$$

where $c>0$ is constant for all beams.

A ground structure made up of beams of uniform cross section may not have enough flexural flexibility to produce a bistable structure. When an arch-shaped structure is loaded from the top (e.g., as in Figs. 2 and 4), the member beams undergo axial as well as bending deformation. The strain energy associated with axial deformation is nonconvex for such structures, while the bending strain energy is convex. Nonconvexity of the total strain energy is typically attained by emphasizing the axial strain energy over the bending strain energy. The role of the thickness modulation is to increase the flexural flexibility, reducing the contribution of the bending to the total strain energy. In order to provide joints with sufficient flexibility, a measure of compliance is achieved by "modulating" the cross section of elements along their axis, reducing the cross sectional area of elements near a flexible joint (alternatively, a soft rubber-like material may be used to model hinges, as illustrated in Sec. 5). In the present work, each member of the ground structure is divided into three regions, as shown in Fig. 7. The two end regions are identical in length $\left(L_{1}\right)$ and cross-

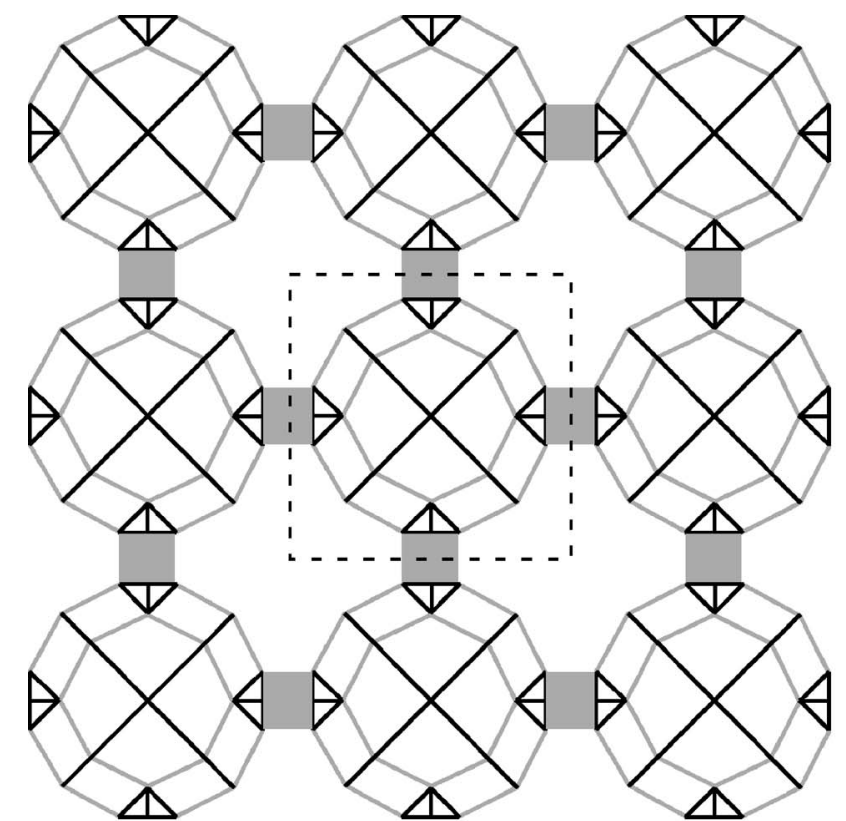

(a)

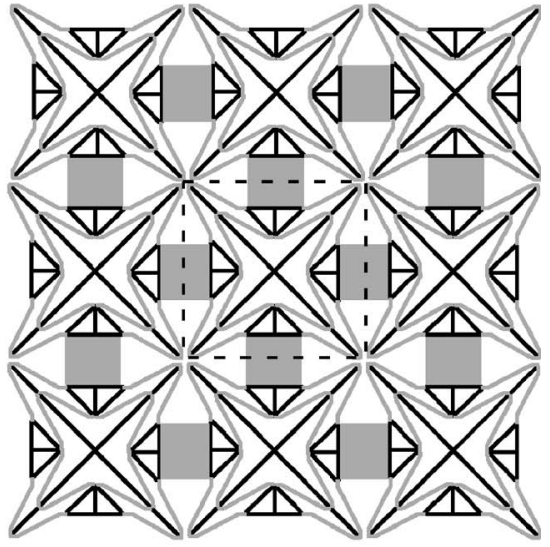

(b)

Fig. 6 A $3 \times 3$ periodic arrangement of bistable structures (a) first equilibrium configuration and (b) second equilibrium configuration 


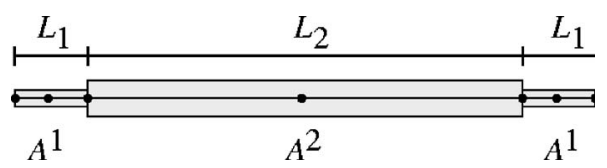

Fig. 7 A member (bar) of the ground structure

sectional area $\left(A^{1}\right)$. The middle region has length $L_{2}$ and crosssectional area $A^{2}$. In a ground structure, $A^{1}$ is an independent (design) variable; we allow $A^{1}$ to take one of three possible values, corresponding to: member removed $\left(A^{1}=0\right)$, member is a snap beam $\left(A^{1}=A^{l}\right)$ or member is a support beam $\left(A^{1}=A^{u}\right)$, where $0<A^{l}<A^{u}$ and $A^{l}$ and $A^{u}$ are prescribed values. $A^{2}$ is a dependent variable, set to 0 (member removed) whenever $A^{1}=0$ and set to $A^{u}$ otherwise. This choice is motivated by the example bistable structure introduced in Sec. 2 which was constructed using only two types of beams: a more compliant one, which snaps and is the source of the bistability, and a stiffer one, which provides support to members of the first type.

Based on the previous discussion, we select as design variables in this problem the cross-sectional areas $A_{\alpha}$ of the end regions of each member $\alpha$ in the ground structure, $\alpha=1,2, \ldots, n_{b}$, and let $A_{\alpha}$ take values in $\left\{0, A^{l}, A^{u}\right\}$.

4.2 The Objective Function. The objective function used here is built around characteristic points of the load displacement curve. A typical curve is shown in Fig. 8. Points $D$ and $F$ correspond to values where the load just reaches the critical value that causes snap-through. Under external loading, $B$ and $H$ are points where the structure settles just after the snap through. Points $C$ and $G$ correspond to the two stable configurations. We use a measure of the distance between these two points to build the objective function. When $\mathbf{x}^{C}$ and $\mathbf{x}^{G}$ are two stable configurations of a simple snap-through structure, this measure is

$$
\phi=\left\|\mathbf{x}^{C}-\mathbf{x}^{G}\right\|^{1 / 2}
$$

In more complex structures, the direction of the snap-through may play a role and to account for this we define

$$
\phi=\sqrt{\left(\mathbf{x}^{C}-\mathbf{x}^{G}\right)^{T} \mathbf{W}\left(\mathbf{x}^{C}-\mathbf{x}^{G}\right)}
$$

where $\mathbf{W}$ is a diagonal, square, semidefinite matrix. Finally, most subsets of the ground structure will not be bistable and in fact, the majority of such subsets will not even be properly formed structures (e.g., they may be disjoined or not properly supported). For any such structure, $\phi$ is set to zero. Thus, the goal achieved by maximizing $\phi$ is simply to find any one bistable structure contained in the ground structure. The function $\phi$ is the objective function to be maximized in the optimization problem, stated in the following section.

Loading a typical external loading is given by

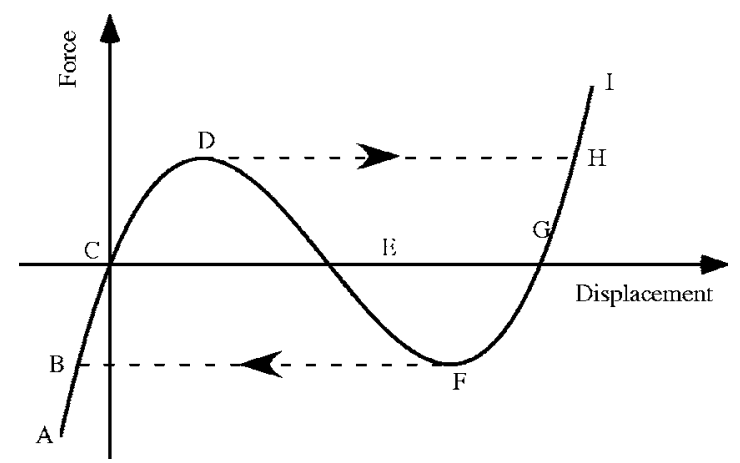

Fig. 8 Typical force-displacement diagram for a bistable structure

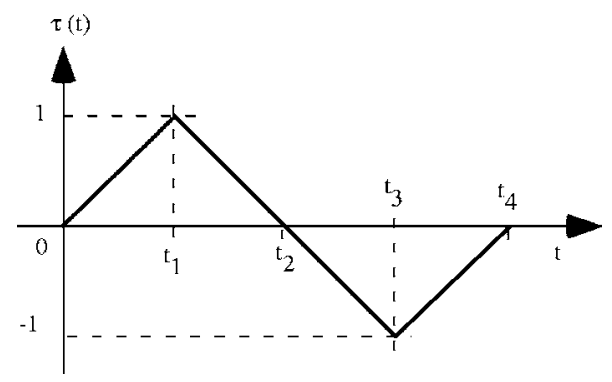

Fig. 9 The external load factor as a function of time

$$
\mathbf{f}_{\text {ext }}(t)=\tau(t) \mathbf{f}^{0}
$$

where $\tau(t)$ is as shown in Fig. 9. $\mathbf{f}^{0}$ is a prescribed maximum external force and $t$ is a pseudotime. The time discretization is chosen to suit the finite element analysis. The goal is to obtain a bistable structure with a critical load less than $\mathbf{f}^{0}$. A displacement driven problem (one where the displacement at the input port is prescribed) is solved to capture the segments $D-E-F-G-H$ and $F$ $E-D-C-B$ of the load-displacement curve (Fig. 8), which are bypassed in a load driven problem. In that case the displacement $x_{i n}$ at the input port(s) is prescribed by

$$
x_{\text {in }}(t)=\tau(t) x_{0}
$$

where $x_{0}$ is a reference displacement amplitude. The force in the ordinate of the force-displacement curve corresponds to the corresponding reaction force.

4.3 The Optimization Problem. The optimization problem is formally stated as

Find $\mathbf{A}=\left\{A_{1}, A_{2}, \ldots, A_{n_{b}}\right\} \in\left\{0, A^{l}, A^{u}\right\}^{n_{b}}$ that

$$
\begin{gathered}
\text { maximize } \phi=\sqrt{\left(\mathbf{x}^{C}-\mathbf{x}^{G}\right)^{T} \mathbf{W}\left(\mathbf{x}^{C}-\mathbf{x}^{G}\right)} \\
\text { subject to } \sum_{\alpha=1}^{n_{b}} \chi\left(A_{\alpha}\right) \leqslant n_{\mathrm{MAX}}
\end{gathered}
$$

where

$$
\chi\left(A_{\alpha}\right)= \begin{cases}0 & \text { if } A_{\alpha}=0 \\ 1 & \text { if } A_{\alpha}>0\end{cases}
$$

Data for this problem are:

- $A^{l}$ : the cross-sectional area of the thin beams;

- $A^{u}$ : the cross-sectional area of the thick beams;

- $n_{\mathrm{MAX}}$ : the maximum number of members allowed; and

- W: prescribed weight factors used to emphasize a given snap-through direction.

4.4 The Solution Scheme. Problem (9) is treated as a combinatorial optimization problem and solved using a genetic algorithm (GA). However, since many, if not most of the subsets of the ground structure are not bistable and may not even be well formed structures, a significant portion of the design space has objective function values $\phi=0$. In order to provide for a meaningful evaluation of the merit of such structures, the original objective $\phi$ in (9) is replaced by the minimization of the following merit function in the GA

$$
f=-(w \phi)^{2}+\psi
$$

In $(10)$

$$
\psi=\psi_{1}+\psi_{2}+\psi_{3}+\psi_{4}
$$

is a penalty function with entries $\psi_{1}, \psi_{2}, \psi_{3}$, and $\psi_{4}$ designed to provide all subsets of the ground structure with a meaningful merit function value (even subsets that are not well formed structures). The (constant) scaling factor $w$ is scaled so that $w \phi \approx 1$, as 


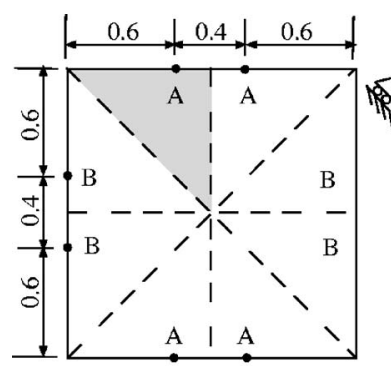

(a)

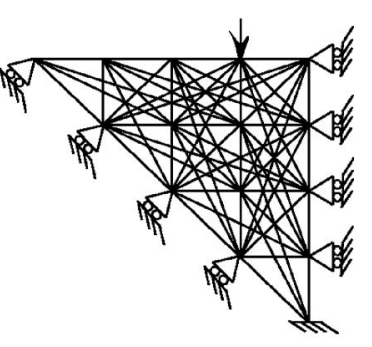

(b)
Fig. 10 Example 1: The package space (a) and corresponding ground structure (b) with dimensions (in $\mathrm{mm}$ ) and boundary conditions

are $\psi_{1}, \psi_{2}, \psi_{3}$, and $\psi_{4}$. These functions are defined as follows:

1. $\psi_{1}$ penalizes structures that do not have any structural members at the prescribed loading ports (nodes). $\psi_{1}$ is defined as

$$
\psi_{1}=\frac{1}{\delta_{\max }} \sum_{j=1}^{n_{f}} \delta_{j}
$$

where $\delta_{j}$ is the distance between the structure and the $j$ th loading port and $\delta_{\max }$ is the maximum distance between two nodes in the structure.

2. $\psi_{2}$ penalizes structures with too many bars. $\psi_{2}$ is defined as

$$
\psi_{2}=\left\{\begin{array}{l}
\frac{\left(n_{a}-n_{\mathrm{MAX}}\right)}{\left(n_{t}-n_{\mathrm{MAX}}\right)} \quad \text { if } n_{a}>n_{\mathrm{MAX}} \\
0 \quad \text { if } n_{a} \leqslant n_{\mathrm{MAX}}
\end{array}\right\}
$$

where

$$
n_{a}=\sum_{\alpha=1}^{n_{b}} \chi\left(A_{\alpha}\right)
$$

is the actual number of members in the structure and $n_{t}$ $>n_{\mathrm{MAX}}$ is a prescribed number used to adjust the slope of the penalty and selected to keep the value of $\psi_{2}$ near 1 (e.g., $n_{t}=2 n_{\mathrm{MAX}}$ ). A lower (or higher) $n_{t}$ increases (or decreases) the relative weight of $\psi_{2}$ with respect to the other $\psi_{i}$ s.

3. $\psi_{3}$ penalizes disjoint structures. $\psi_{3}$ is defined as

$$
\psi_{3}=\frac{N_{s}}{N_{t}}
$$

where $N_{s}$ is the number of disjoint substructures and $N_{t}$ is a prescribed scaling factor (e.g., $N_{t}=2$ ). To measure $N_{s}$ the structure is represented as an undirected graph. $N_{s}$ is the number of components of the graph. The algorithm (pseudocode) used for computing $N_{s}$ is given in the Appendix.

4. $\psi_{4}$ penalizes structures that are not properly supported, i.e., structures that may undergo rigid-body motions. $\psi_{4}$ is defined as

$$
\psi_{4}=N_{x}+N_{y}+N_{\theta}
$$

where $N_{x}=0$ if the structure's rigid-body motion in the $x$ direction is constrained, $N_{x}=1$ otherwise, and similarly for $N_{y}$ and $N_{\theta}$. Only a straight forward evaluation is made, simple enough to rule out designs with little computation. For instance, $N_{x}$ is set to 0 if a node connected to the structure is on an $x$-constrained boundary.

\section{Examples}

5.1 Example 1. In this example the goal is to design a twodimensional bistable structure that will operate, loaded at ports $A$

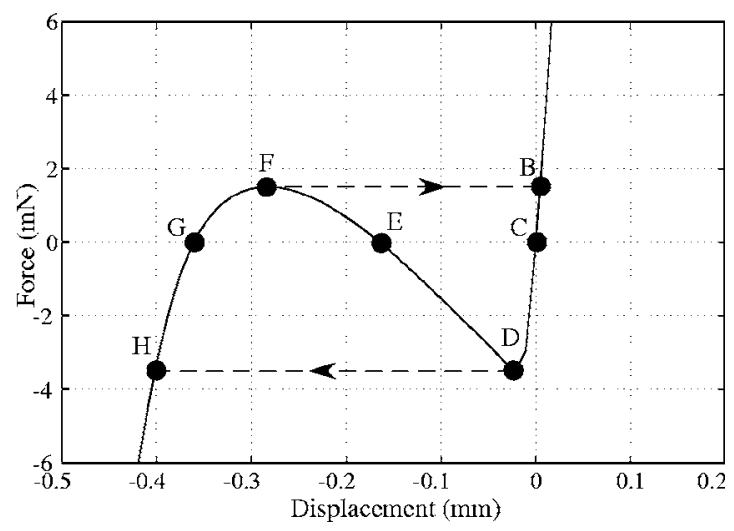

Fig. 11 The force-displacement diagram for the reference structure (Fig. 3)

and $B$ and fit within a $1.6 \mathrm{~mm} \times 1.6 \mathrm{~mm}$ package space, as shown in Fig. 10. This space is partitioned into eight triangles, whose boundaries are marked with dashed lines in the figure. The structure is assumed to be symmetric about these lines. The ground structure is laid on one of these triangles and the appropriate boundary conditions are applied to enforce symmetry (Fig. 10(b)). Loads $F=6 \tau(t)$ in the range $\pm 6 \mathrm{mN}$ are applied at positions $A$ and $B$. The function $\tau(t)$ is as in Fig. 9. All entries in the weight matrix W are zero except $W_{m m}=1$, where $m$ is the degree-of-freedom associated with the load $F$. This means that we want to maximize snap-through in the direction of the external load.

The total number of bars in the ground structure $\left(n_{b}\right)$ is 62 . In accordance with Sec. 4.1, every member is discretized into three regions with lengths $L_{2}=6 L_{1}$ and every region is further discretized into two finite elements (Fig. 7). A solution is allowed a maximum of $n_{\mathrm{MAX}}=8$ bars, each using one of two cross section areas: $A^{l}=10^{-4} \mathrm{~mm}^{2}$ and $A^{u}=10^{-3} \mathrm{~mm}^{2}$. The Young's modulus and Poisson's ratio are $1380 \mathrm{MPa}$ and 0.3 , respectively.

The ground structure depicted in Fig. 10(b) admits, as one solution, the topology of the bistable structure introduced in Sec. 2 (Fig. 3), the reference structure. That structure has an objective function $\phi=0.3674(\mathrm{~mm})$ (we use this value to scale the objective function $f$ in (9) and define $w=1 / 0.3674=2.7218$ ). The structure has the force displacement diagram shown in Fig. 11. The switching force (the force that causes snap-through) for this mechanism is approximately $-3.5 \mathrm{mN}$ in the forward direction and $1.8 \mathrm{mN}$ in the backward direction.

We now use a GA to find other solutions. The GA starts with a population of 100 randomly generated designs, which are sorted based on their merit function $(f)$ values. Following a standard GA procedure, every individual is assigned a scaled merit function value depending on its position. An elitist strategy with generation

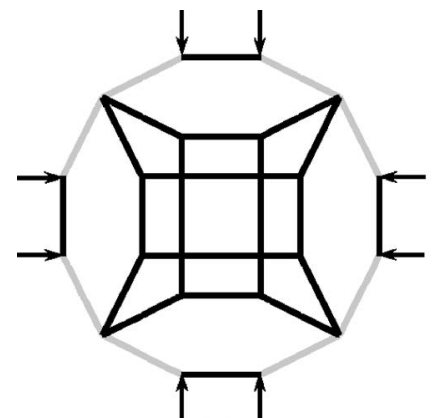

(a)

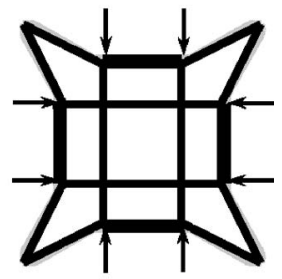

(b)
Fig. 12 Example 1: Bistable structure obtained by the GA (a) first stable configuration and $(b)$ second stable configuration 


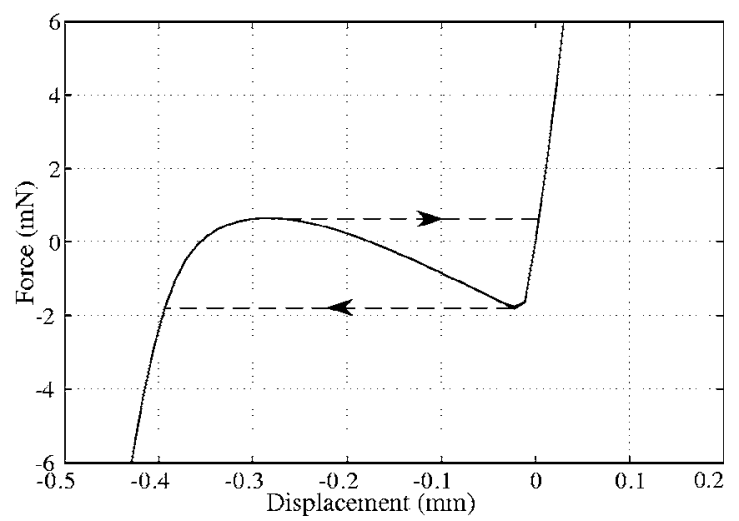

Fig. 13 The force-displacement diagram for the structure in Fig. 12

gap of $60 \%$ is used. The best $40 \%$ of the solutions in the population are carried forward to the next generation and the remaining $60 \%$ is produced through crossover among the parents, selected probabilistically from within the current generation. The probabilistic selection of parents for crossover is based on the standard "roulette wheel" mechanism, where the probability of selection of an individual for the crossover is directly proportional to its fitness value. The mutation rate used is $10 \%$.

The process of fitness assignment, crossover, mutation etc. was repeated for 500 generations, which resulted in the solution shown in Fig. 12. The objective function value for this structure is $\phi$ $=0.3688(\mathrm{~mm})$, with penalty function $\psi=0$, i.e., only slightly better than the structure in Fig. 3. The corresponding force displacement diagram (obtained with the prescribed input displacement) is shown in Fig. 13. The switching force is approximately $-1.8 \mathrm{mN}$ in one direction (forward) and $0.9 \mathrm{mN}$ in the other (backward).

Another solution with essentially the same objective function value (and $\psi=0$ ) is shown in Fig. 14. In this structure, however, several members overlap. The present modeling does not account for interference or contact between members, as the structure switches from one configuration to the other. This is acknowledged as a drawback, unfortunately one that is difficult to overcome. This will be discussed further in the next section.

Solutions in Figs. 12 and 14 show that the deformation in the hinges can be quite large, raising questions regarding the manufacturability of solutions modeled using these ideas. Some of these concerns may be alleviated by using a rubber-like material (polyurethane elastomer) at the hinges. The maximum allowable normal stress in this material is about $45 \mathrm{MPa}$, which corresponds to about $440 \%$ elongation at break. For instance, returning to the structure shown in Fig. 14, one can use the soft material in the two end sections of each hinged member (shown light gray in the figure) and keep the area constant at $A^{u}$ over the length of the member. This would result in the load-displacement curve shown in Fig. 15. Note that using the rubber-like material does not

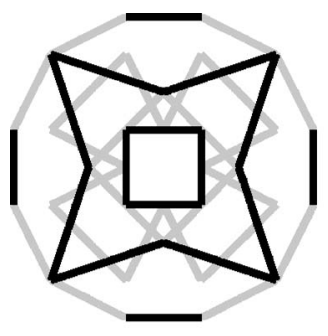

(a)

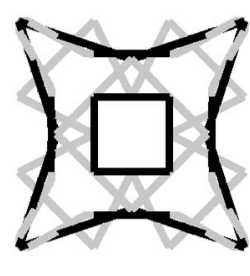

(b)
Fig. 14 Example 1: Another bistable structure (a) first stable configuration and $(b)$ second stable configuration

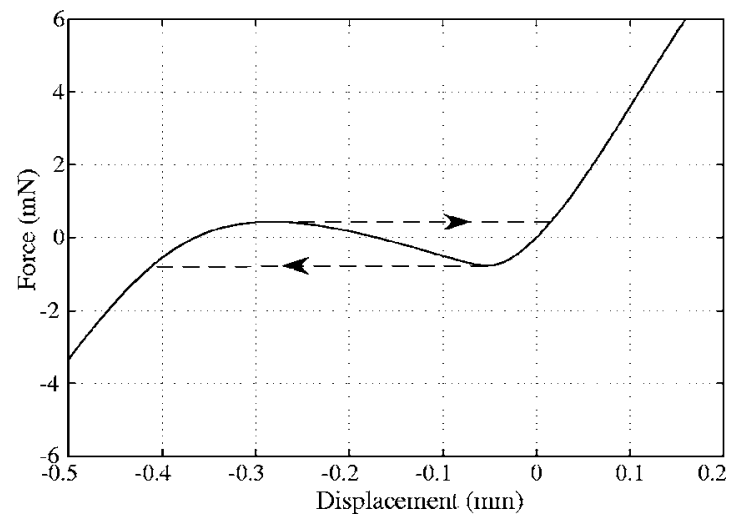

Fig. 15 The force-displacement diagram for the structure in Fig. 14 with polyurethane elastomer hinges

change the size of the jump between stable configurations significantly (but it does change the magnitude of the actuating force required to switch configurations). The maximum stress in the elastomer is $25.5 \mathrm{MPa}$, within the allowable limit for the material. This suggests that using a combination of materials that includes softer materials near the hinges may result in concepts that could be manufactured more easily.

All the solutions shown here are obtained from the same run of the GA. Figure 16 shows the history of the best GA merit function $(f)$ value for a generation. At generation 0, i.e., in the randomly generated population, the best merit function value is $f=0.1250$. At the end of 500th generation, the best merit function value is $f=-1.008$. The finite element analysis and optimization procedures were implemented on a MATLAB program running on a Pentium 4 machine with $2.8 \mathrm{GHz}$ clock speed, $496 \mathrm{MB}$ random access memory and Windows XP operating system. $93 \mathrm{~h}$ were spent in running the optimization program for 500 generations.

5.2 Example 2. The package space in this example is the same as that in example 1. The goal is to maximize the snapthrough at ports $B$ in the horizontal direction, when the structure is loaded (only) at ports $A$, in the vertical direction.

A load $F$ in the range of $\pm 4 \mathrm{mN}$ is applied at each loading port. With the given loading, the structure is assumed to be symmetric about the horizontal and vertical centerlines, which are shown as dashed lines in Fig. 17(a). In addition, we want a structure to be orthotropic. For this reason, the layout is assumed to be symmetric about the diagonals, shown as thin dashed lines in Fig. 17(a). Accordingly, the package space is partitioned into eight equal triangles, whose boundaries are marked with dashed lines in the figure. The ground structure is laid on one of these triangles. The ground structure is the same as that in example 1, as are other

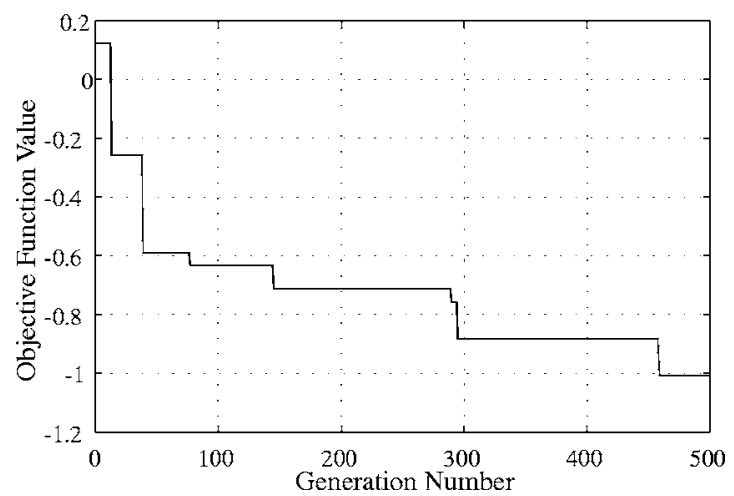

Fig. 16 History of the best GA merit function value $f$ 


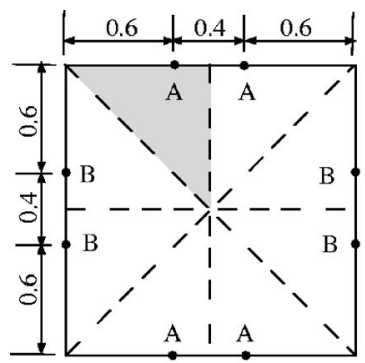

(a)

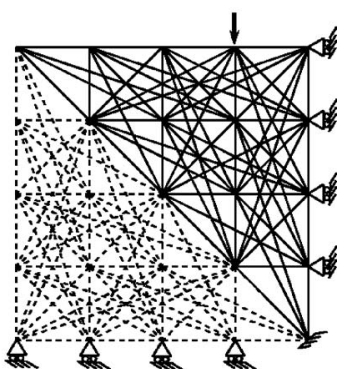

(b)
Fig. 17 Example 2: the package space (a) and corresponding ground structure $(b)$ with dimensions (in $\mathrm{mm}$ ) and boundary conditions

details of the discretization. It should be noted, however, that since the loading is symmetric only about the centerlines, one quarter of the structure is analyzed (Fig. 17(b)). Appropriate boundary conditions are applied to enforce symmetry, as shown in the figure.

In this example all entries in the weight matrix $\mathbf{W}$ are zero except $W_{p p}=1$, where $p$ is the degree-of-freedom associated with the horizontal motion of the output port $B$. The GA parameters such as population size, mutation rate and generation gap have been kept as in example 1. The GA was run for 500 generations, after which a solution is obtained with merit function value $f=$ $-0.10614, \phi=0.1197(\mathrm{~mm})$, and $\psi=0$. The solution is shown in Fig. 18(a). Figure 18(b) shows the corresponding second stable configuration of the structure.

The external force at input port $A$ is plotted versus the displacement at the output port $B$ in Fig. 19 (obtained from a displacement-driven analysis). The maximum switching forces are approximately $-1.9 \mathrm{mN}$ in the forward direction and $0.5 \mathrm{mN}$ in the backward direction. The maximum switching force in the backward direction is small compared to that in the forward direction. While the present model does not penalize this difference, control of the relative magnitudes of the switching forces can be accommodated in the GA formulation by simply adding a new penalty term to the objective function.

In Fig. 19 we notice that the displacement at the output port $B$ does not change sign through the input cycle, i.e., as $\tau(t)$ (Fig. 9) goes through one full cycle. The force-displacement curve is nearly vertical in the neighborhood of the first stable configuration, suggesting that in this region the output port remains nearly stationary for a small range of inputs, a behavior reminiscent of a Poisson's ratio zero material. This is of course not true in a neighborhood of the second stable configuration, where a small external force applied at the input port makes the output port $B$ move a finite distance.

Finally, again we observe in the solution proposed to example 2

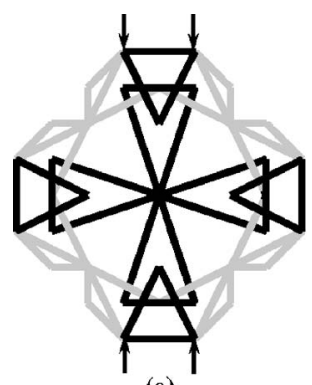

(a)

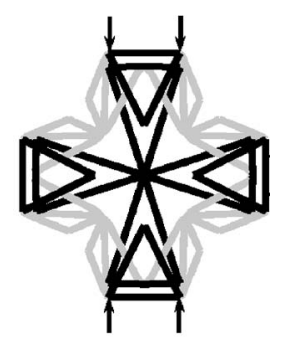

(b)
Fig. 18 Example 2: bistable structure obtained by the GA (a) first stable configuration and $(b)$ second stable configuration

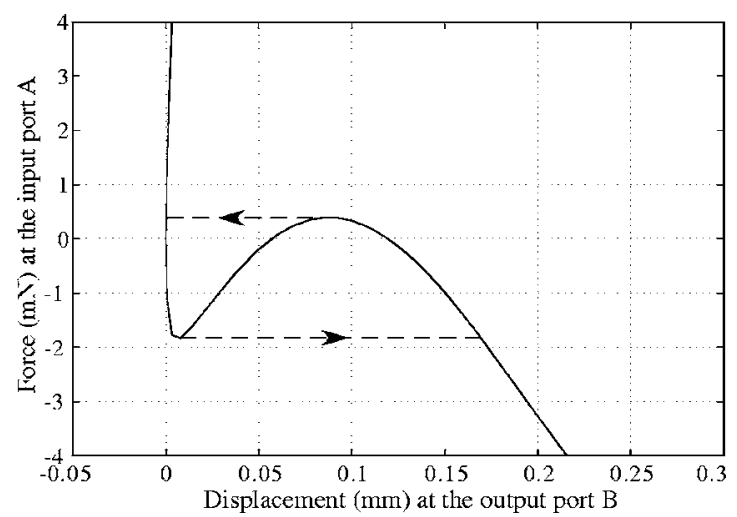

Fig. 19 Example 2: plot of the internal force at the input port $A$ versus the displacement at the output port $B$

that several members overlap as the structure moves from one configuration to the other, signaling interference. In this problem, however, we were unable to find a solution where interference did not occur. One way to address this problem is to cast the problem in three dimensions, allowing at least some members to have a small curvature out of the plane, to avoid interference. This can be appreciated in Fig. 20, which shows quarter sections of the solution in its two stable configurations. Here, as in all previous solutions, one can identify three main substructures. One substructure, formed by stiff members, remains fixed in space throughout the deformation and acts as support to the rest of the structure. A second substructure moves essentially as a rigid body and is also formed by stiff members. This substructure typically receives input from or transfers output to neighboring components. The third substructure connects the previous two and is formed by compliant members. This substructure is primarily responsible for the motion of the mechanism. In most cases interference is avoided if these three components are in different planes of the structure. This qualitative description provides hints on how to actually implement these solutions. Naturally, this is not sufficient in many applications, e.g., in very small scale designs where a layered solution is not realistic. In such cases a full implementation of a contact detection scheme that rejects solutions where interference is detected would be necessary.

5.3 Example 3. Similar to example 2, the goal in this example is to maximize the snap-through at the output ports $B_{1}$ and $B_{2}$ in the horizontal direction, when the structure is loaded at ports $A_{1}$ and $A_{2}$ in the vertical direction (Fig. 21(a)). The package space in this example is a $1.2 \mathrm{~mm} \times 1.2 \mathrm{~mm}$ square. A load $F$ in the

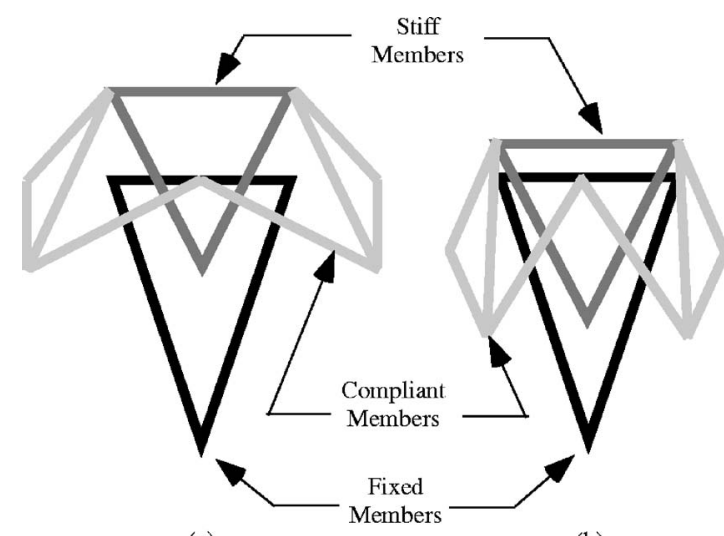

(a)

(b)

Fig. 20 Detail of the solution of Example 2: (a) first stable configuration and $(b)$ second stable configuration 


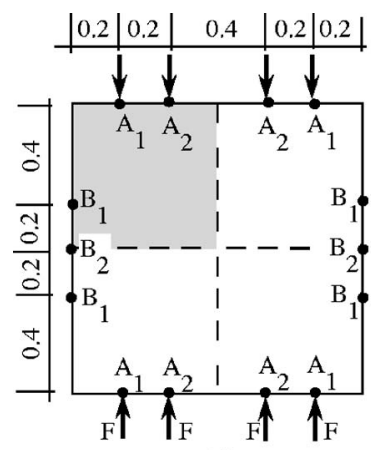

(a)

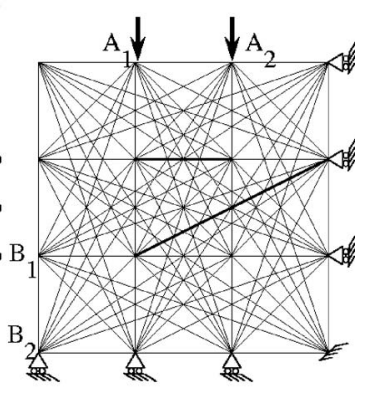

(b)
Fig. 21 Example 3: the package space (a) and corresponding ground structure $(b)$ with dimensions (in $\mathrm{mm}$ ) and boundary conditions

range of $\pm 3 \mathrm{mN}$ is applied at each loading port.

Here we study the effect of a change in the ground structure, which is now as shown in Fig. 21(b). The structure is assumed to be symmetric about the horizontal and vertical centerlines (shown as dashed lines in Fig. 21(a)) but not about the diagonals. The package space is partitioned into four equal squares, whose boundaries are marked by dashed lines in the figure. The ground structure (Fig. 21(b)) is laid on one of these squares. Appropriate boundary conditions are applied to enforce symmetry. The total number of bars in the ground structure $\left(n_{b}\right)$ is 86 . A solution is allowed a maximum of $n_{\mathrm{MAX}}=12$ bars.

In this example all entries in the weight matrix $\mathbf{W}$ are zero except $W_{q q}=1 / 2$, where $q$ is the degree-of-freedom associated with the horizontal motion of the output ports. The GA parameters (such as population size, mutation rate and generation gap) and material and section properties have been kept as in example 1 .

The GA was run for 500 generations, after which a solution is obtained with merit function value $f=-0.2817, \phi=0.1950(\mathrm{~mm})$, and $\psi=0$. The solution is shown in Fig. 22(a). Figure 22(b) shows the corresponding second stable configuration of the structure. Note that in the solution to this example the output ports move outwards while in the solution to example 2 they move inwards (recall that the objective function is indifferent to the directionleft or right—of the snap)

Figure 23 shows the total internal force $(2 F)$ at input ports plotted versus the average displacement at the output ports. The displacements at the output ports $B_{1}$ and $B_{2}$ are essentially equal throughout the analysis. The maximum total switching force is approximately $-3.8 \mathrm{mN}$ in the forward direction and $4.2 \mathrm{mN}$ in the backward direction.

Finally, note that also in this problem the solution is formed by fixed, movable rigid-body and flexible substructures, as in the previous examples. Overlapping of these substructures does not pose a problem, as these substructures can actually be joined together without hampering the performance of the bistable structure.

\section{Conclusions}

The model presented is quite simple. It ignores important constraints, such as interference, contact between members, and stress constraints. The simplifications introduced were necessary to reduce the computational burden associated with the analysis and, in particular, with the use of genetic algorithms. However, in spite of its simplicity, the approach results in a useful tool to explore concepts in design of bistable, periodic structures. Further studies should include a better objective function, capable of providing sensitivity information even for the monostable designs; the computation of analytical sensitivity information, consistent with more efficient, gradient based optimization algorithms; stress and inter-

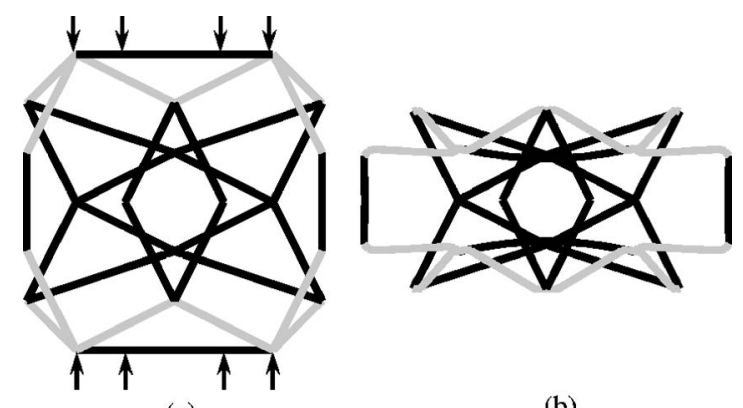

(a)

(b)

Fig. 22 Example 3: bistable structure obtained by the GA (a) first stable configuration and $(b)$ second stable configuration

ference constraints; and more refined analysis models (e.g., twodimensional elasticity instead of beam models) that may reflect the true behavior of compliant structures more accurately.

Essentially all solutions found work on a similar principle: they exploit the bistability of simple 2- or 3-bar segments placed strategically around a supporting frame. We do not see this as a reason for concern but, in fact, as insight that can be exploited to develop different and perhaps more efficient design strategies. For example, with this insight one could devise a different algorithm, perhaps one in which the design space is represented not by means of a ground structure, but instead by a vocabulary that involves only 2 bar substructures and structures to support them (and a grammar that works on connecting pieces to each other and to the external loads). This could be a promising new approach, to be pursued in future work.

\section{Acknowledgment}

This work was sponsored, in part, by Toyota Motor Corporation. Their support is gratefully acknowledged.

\section{Appendix} (15):

The following algorithm (pseudocode) is used to compute $N_{s}$ in

Step 1:

Step 2:

Step 3:

Step 4:

Step 5:

Step 6:

Step 7:

Step 8:

justVisitedEle $=\varnothing$

RemainingElements $=$ ElementList

nStruct $=0$

While RemainingElements $\neq \varnothing$, Do Steps $5-10$

IsExhausted $=$ False

gateNodes $=$ Nodes $($ RemainingElements $(1))$

While IsExhausted $=$ False, Do Steps 8 and 9

justVisitedEle $=$ RemainingElements $\cap$

Elements(gateNodes)

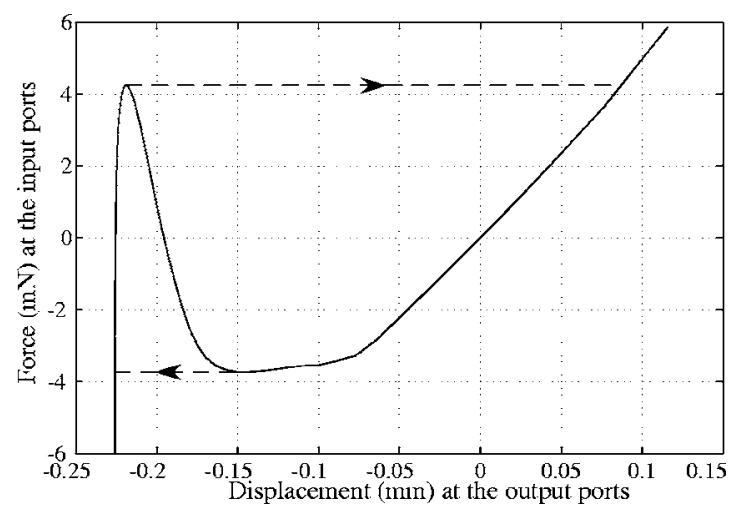

Fig. 23 Example 3: plot of the total internal force at the input ports versus the average displacement at the output ports 
Step 9:

$$
\text { If justVisitedEle }=\varnothing
$$$$
\text { IsExhausted }=\text { true }
$$

else

gateNodes $=$ Nodes(justVisitedEle $)$ - gateNodes RemainingElements $=$ RemainingElements justVisitedEle

Step 10: $\quad$ nStruct $=$ nStruct +1

Step 11: $\quad N_{s}=$ nStruct -1

where

- ElementList is the set of all element numbers in the structure.

- Nodes is a function which takes element number as an argument and gives the two node numbers connected by the element.

- Elements is a function which takes node number as an argument and gives all the elements connected at that node.

- RemainingElements(1) refers to the first member of RemainingElements.

\section{References}

[1] Wang, Y. C., and Lakes, R. S., 2004, "Extreme Stiffness Systems Due to Negative Stiffness Elements," Am. J. Phys., 72(1), pp. 40-50.

[2] Jensen, B. D., Howell, L. L., and Salmon, L. G., 1999, "Design of Two-Link, In-Plane, Bistable Compliant Micro-Mechanisms," ASME J. Mech. Des., 121, pp. 416-423.

[3] Jensen, B. D., Parkinson, M. B., Kurabayashi, K., Howell, L. L., and Baker, M. S., 2001, "Design Optimization of a Fully-Compliant Bistable MicroMechanism," Proceedings of the ASME International Mechanical Engineering Congress and Exposition, IMECE2001/MEMS-23852, New York.

[4] Jensen, B. D., and Howell, L. L., 2003, "Identification of Compliant PseudoRigid-Body Four-Link Mechanism Configurations Resulting in Bistable Behavior," ASME J. Mech. Des., 125, pp. 701-708.

[5] Jensen, B. D., and Howell, L. L., 2004, "Bistable Configurations of Compliant Mechanisms Modeled Using Four Links and Translational Joints," ASME J. Mech. Des., 126(4), pp. 657-666.

[6] Masters, N. D., and Howell, L. L., 2003, “A Self-Retracting Fully-Compliant Bistable Micromechanism,” J. Microelectromech. Syst., 12, pp. 273-280.
[7] Masters, N. D., and Howell, L. L., 2005, "A Three Degree-of-Freedom Model for Self-Retracting Fully Compliant Bistable Micromechanisms," ASME J. Mech. Des., 127(4), pp. 739-744.

[8] King, C., and Campbell, M. I., 2004, "On the Design Synthesis of Multistable Equilibrium Systems," Proceedings of the International Design Engineering and Technical Conference, Salt Lake City, Utah.

[9] Howell, L. L., 2001, Compliant Mechanisms, Wiley, New York.

[10] Ananthasuresh, G. K., and Howell, L. L., 2005, "Mechanical Design of Compliant Microsystems-A Perspective and Prospects," ASME J. Mech. Des., 127(4), pp. 736-738.

[11] Ananthasuresh, G. K., and Kota, S., 1995, "Designing Compliant Mechanisms," Mech. Eng. (Am. Soc. Mech. Eng.), 117(11) pp. 93-96.

[12] Sigmund, O., 1997, "On the Design of Compliant Mechanisms Using Topology Optimization," Mech. Struct. Mach., 25, pp. 493-524.

[13] Frecker, M. I., Ananthasuresh, G. K., Nishiwaki, S., Kikuchi, N., and Kota, S., 1997, "Topological Synthesis of Compliant Mechanisms Using Multi-Criteria Optimization," ASME J. Mech. Des., 119(2) pp. 238-245.

[14] Tai, K., and Chee, T. H., 2000, "Design of Structures and Compliant Mechanisms by Evolutionary Optimization of Morphological Representations of Topology," ASME J. Mech. Des., 122(4), pp. 560-566.

[15] Bendsøe, M. P., and Kikuchi, N., 1988, "Generating Optimal Topologies in Structural Design Using a Homogenization Method," Comput. Methods Appl. Mech. Eng., 71, pp. 197-224.

[16] Bruns, T. E., Sigmund, O., and Tortorelli, D. A., 2002, "Numerical Methods for the Topology Optimization of Structures that Exhibit Snap-Through," Int. J. Numer. Methods Eng., 55, pp. 1215-1237.

[17] Saxena, A., and Ananthasuresh, G. K., 2001, "Topology Synthesis of Compliant Mechanisms for Nonlinear Force-Deflection and Curved Path Specifications," ASME J. Mech. Des., 123(1), pp. 33-42.

[18] Joo, J. Y., and Kota, S., 2004, "Topological Synthesis of Compliant Mechanisms Using Nonlinear Beam Elements," Mech. Based Des. Struct. Mach., 32(1), pp. 17-38.

[19] Maddisetty, H., and Frecker, M., 2004, "Dynamic Topology Optimization of Compliant Mechanisms and Piezoceramic Actuators," ASME J. Mech. Des., 126(6), pp. 975-983.

[20] Saxena, A., 2005, "Synthesis of Compliant Mechanisms for Path Generation Using Genetic Algorithm,” ASME J. Mech. Des., 127(4), pp. 745-752.

[21] Qiu, J., Lang, J., and Slocum, A. H., 2004, "A Curved-Beam Bistable Mechanism,” J. Microelectromech. Syst., 13(2), pp. 137-146.

[22] Crisfield, M. A., 1991, Non-Linear Finite Element Analysis of Solids and Structures, Wiley, Chichester, Vol. 1.

[23] Dorn, W. C., Gomory, R. E., and Greenberg, H. J., 1964, “Automatic Design of Optimal Structures," J. Mec., 3, pp. 25-52.

[24] Bendsøe, M., Ben Tal, A., and Zowe, J., 1994, "Optimization Methods for Truss Geometry and Topology Design,” Struct. Optim., 7, pp. 141-159. 